

Wang, D., Zhang, L., Qi, Y. and Quddus, A. U. (2015) Localized Mobility Management for SDN-Integrated LTE Backhaul Networks. In: 2015 IEEE 81st Vehicular Technology Conference (VTC Spring), Glasgow, Scotland, 11-14 May 2015, ISBN 9781479980888.

There may be differences between this version and the published version. You are advised to consult the publisher's version if you wish to cite from it.

http://eprints.gla.ac.uk/143644/

Deposited on: 6 July 2017

Enlighten - Research publications by members of the University of Glasgow http://eprints.gla.ac.uk 


\title{
Localized Mobility Management for SDN-Integrated LTE Backhaul Networks
}

\author{
Dongyao Wang, Lei Zhang, Yinan Qi, Atta Ul Quddus \\ Institute for Communication Systems \\ University of Surrey \\ Guildford, Surrey, GU2 7XH, United Kingdom \\ Email: \{w.dongyao, lei.zhang, yinan.qi, a.quddus\}@surrey.ac.uk
}

\begin{abstract}
Small cell (SCell) and Software Define Network (SDN) are two key enablers to meet the evolutional requirements of future telecommunication networks, but still on the initial study stage with lots of challenges faced. In this paper, the problem of mobility management in SDN-integrated LTE (Long Term Evolution) mobile backhaul network is investigated. An 802.1ad double tagging scheme is designed for traffic forwarding between Serving Gateway (S-GW) and SCell with QoS (Quality of Service) differentiation support. In addition, a dynamic localized forwarding scheme is proposed for packet delivery of the ongoing traffic session to facilitate the mobility of UE within a dense SCell network. With this proposal, the data packets of an ongoing session can be forwarded from the source SCell to the target SCell instead of switching the whole forwarding path, which can drastically save the path-switch signalling cost in this SDN network. Numerical results show that compared with traditional path switch policy, more than $50 \%$ signalling cost can be reduced, even considering the impact on the forwarding path deletion when session ceases. The performance of data delivery is also analysed, which demonstrates the introduced extra delivery cost is acceptable and even negligible in case of short forwarding chain or large backhaul latency.
\end{abstract}

Index Terms-small cell; software defined network; mobility management; traffic forwarding

\section{INTRODUCTION}

Data traffics are experiencing an exponential increase due to the fast growing population of smart phones and mobile applications. This trend will continue over the next decade with a common consensus in the wireless industry that an astounding 1000-fold increase in data traffic would be supported [1][2][3]. This rapid growing need has resulted in tremendous advances in mobile networks both at the radio access and the network management. In this regard, the technology of SCell is the most promising frontier to drive wireless capacity growth due to the increased spectrum reuse and improved spectrum efficiency [2]. However, the SCell deployment results in a larger number of base stations that require a backhaul network with higher connectivity, which calls for a simplification of the mobile access network.

An emerging technology for supporting flexibility in networking is software defined networking [4], which provides an abstraction between data-plane forwarding hardware and control software. In this way, a logical centralized SDN controller allows to program the network on a per-flow basis with some protocols, among which OpenFlow is the most popular one and is currently being standardized by ONF (Open Network Foundation) organization [4]. The SDN and OpenFlow are originally designed for wired network, and now are being extended to mobile network [5][6][7][8][9], i.e. Software Defined Mobile Networks (SDMN). One study [6] described a generic SDWN architecture which bring the benefits of logical orchestration and flexibility of traffic handling. In FP7 project CROWD (Connectivity management for eneRgy Optimised Wireless Dense networks)[7], SDN is identified as a solution to tackle extreme density of wireless networks. Another work [9] studied the solution to virtualize EPC (Evolved Packet Core) architecture with data center and handle data traffics via SDNbased switches.

Different from previous works which focused on architecture design of SDMN, here we will study the problem of mobility management within the SDN framework. Mobility management is a critical aspect of mobile networks which imposes lots of challenges on network architectures, especially in case of dense SCell deployment scenarios [8][10]. In 3GPP (The 3rd Generation Partnership Project) LTE Advanced network architecture, mobility management is strongly dependent on the reconfiguration of GTP (GRPS Tunneling Protocol) tunnels between access and core network entities. However, due to frequent handover events in the dense SCell deployment scenario, the reconfiguration of GTP tunnels will cause significant signalling load to the core networks entities [12]. Furthermore, excessive reconfigurations of GTP tunnels may degrade the user's quality of experience (QoE).

In addition, there is already some work considering the issue of mobility management under SDN framework. For example, [10] designed a distributed hierarchical architecture based on OpenFlow to enable the mobility management among different radio access networks. [8] suggested to integrate both SDN controller and EPC entities into a single network element to simplify the network functionality. As described in [8], with SDN adoption on the EPC backhaul network, the GTP tunnel can be removed to reduce the transport overhead, but the signalling overwhelming problem for the dense SCell network still exists because of the centralized mode of SDN controller. In a typical SDN network, all the switches are controlled by the SDN controller in a centralized manner, which means all the data-path switches to support UE mobility will require OpenFlow based signalling interaction between the transport 
switches and SDN controller. Thus, it just shifts the signalling problem from MME (Mobility Management Entity) to SDN controller, while the QoE problem is still degraded, just the same as without SDN.

To deal with the above deficiencies, we designed a ne framework for mobility management in SDN-integrated LT mobile backhaul network, where the backhaul network br tween S-GW and SCell is implemented as an SDN networ] Here an 802.1ad VLAN (Virtual LAN) tunneling scheme applied for the delivery of data packet in this SDN networ] Also a dynamic localized forwarding scheme is propose to facilitate UE mobility within a dense SCell networ] The preliminary numerical analysis shows that the proposed scheme can significantly reduce the signalling cost and relieve the processing burden of SDN controller compared to the traditional scheme.

The rest of this paper is structured as follows. Section II describes the SDN-integrated LTE backhaul network and related delivery procedure. In section III, the proposed localized forwarding scheme is presented in detail. The analytical model is developed in section IV with numerical results in section V. Finally, the main conclusions are summarized in section VI.

\section{SDN-INTEGRATED LTE BACKHAUl NeTWORK}

In this paper, for the sake of compatibility with current LTE EPC architecture, the SDN is integrated on the backhaul network, which is designed to control the data plane between SCell eNodeB and S-GW, as shown in Fig. 1. There is an interface between SDN controller and MME, thus the SDN controller can receive mobility events directly from MME. Actually, to further simplify the network functionality, the SDN controller can be integrated with MME into a single network entity while still keeping current MME interface. In this architecture, SCell eNodeB, S-GW and all the intermediate nodes can be regarded as OpenFlow-enaabled switches, which means that the SDN controller can add, modify or delete the flow entries in these switches with OpenFlow protocol.

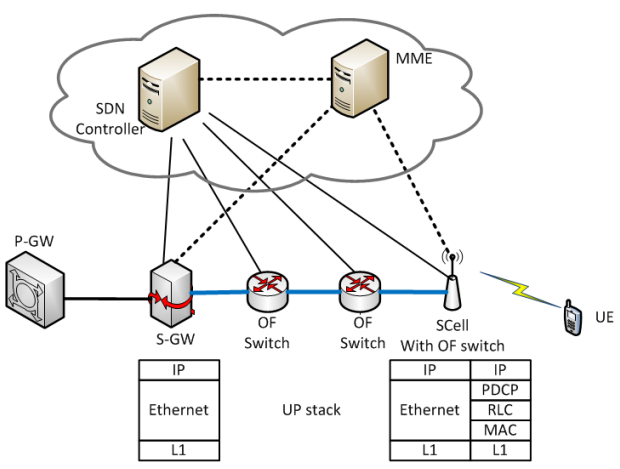

Fig. 1. SDN-integrated Architecture

In addition, the data forwarding in this SDN network is implemented at Layer 2. The obvious benefit is to simplify the stack of user data plane on both SCell and S-GW as shown in Fig. 1. SCell will terminate the radio layers and support Ethernet switch towards the backhaul network, and SGW consists of simple Ethernet switch and IP router towards P-GW (Packet Data Network Gateway) or public Internet.

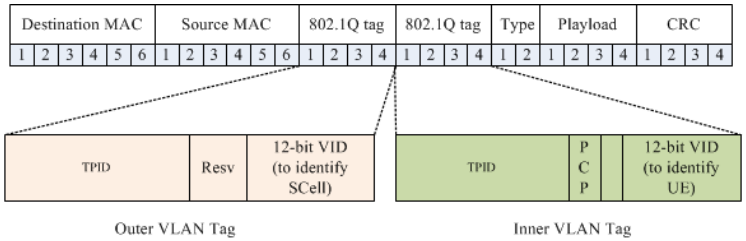

Fig. 2. Used 802.1ad Ethernet Frame

802.1ad with double VLAN tagging is introduced in this SDN integration to support different traffic flow with different QoS[8][13]. Thus as shown in Fig. 2, the frame packets delivered on this SDN backhaul network have the structure of Ethernet 802.1ad VLAN format. The SDN controller will use two VLAN taggings to configure the flow entries and optimize the forwarding path in the backhaul network. The outer VLAN tagging has only one effective field, i.e. 12-bit VID (VLAN identifier) which is used to identify one SCell, and is allocated by SDN controller when the SCell joins the network. Other fields can be reserved for future purpose. Obviously, such an SDN network can support maximal 4094 SCells (VID 0x000 and $0 \mathrm{xFFF}$ are reserved). The inner VLAN tagging has two meaningful parts in this design, where 3-bit PCP (Priority Code Point) indicates the QoS information of flow and 12bit VID for the identification of UE. Thus maximal 4094 UEs (VID 0x000 and 0xFFF are also reserved) are supported in each SCell. When a UE enters ECM-CONNECTED mode, the SDN controller will allocate a VID for this UE and use it to configure the flow entries in the SDN backhaul network. On the contrary, when a UE enters ECM-IDLE mode, the allocated VID will be reclaimed for future allocation. In addition, when a UE handover from source SCell to target SCell, it will be allocated a new VID in target SCell domain with its old VID reclaimed by source SCell domain. It is the responsibility of SDN controller to guarantee the uniqueness of allocated VIDs within a SCell.

\section{LOCALIZED ForWARDING SCHEME For HANDOVER}

In this architecture the mobility management is performed by the SDN controller. According to traditional policy, when a UE moves from source SCell to target SCell, the SDN controller will take the responsibility to update the forwarding path for ongoing sessions belonging to this UE, i.e. the SDN controller may need to add new flow entries on the new forwarding path between S-GW and the target SCell, and delete useless flow entries on the old forwarding path between $\mathrm{S}-\mathrm{GW}$ and the source SCell. As described in section I, this scheme would introduce overwhelming signalling overhead due to frequent handovers in the dense SCell deployment scenario. With this in mind, considering the scenario where the SCell has direct or indirect Ethernet connections with its neighboring cells, we propose a dynamic localized mobility management method in this SDN-integrated backhaul network. 
Instead of switching the forwarding path after handover, the previous forwarding path will be maintained for the ongoing session communication. With handover procedure completed, the traffic data can be forwarded from source SCell to target SCell via existing Ethernet connection. Thus a traffic forwarding chain between SCells may be established from the original serving SCell to the current serving SCell. To implement the above scheme, the following four operations are required:

(1) When a UE handovers from source SCell to target small, it will be allocated a new VID as usual by SDN controller and the VID is unique within the domain of target SCell. Different from section II, the old VID will not be reclaimed in source SCell domain until the ongoing session ceased.

(2) After handover, the SDN controller will still control to forward traffic data of the UE along the previous path from S-GW to the source SCell, by using doubling VLAN tagging with the VID of source SCell and old VID of the UE in the domain of source SCell.

(3) The SDN controller will modify the flow entry in source SCell to update the double VLAN tagging of the arriving packets belonging to the UE, with the VID of target SCell and new allocated VID of the UE in the domain of target SCell, then forward them to the target SCell.

(4) Finally, when the target SCell receives these packets, it will remove the double tagging of these packets and then send them to upper layer, i.e. IP layer. Certainly all these procedures are under the control of SDN controller.

This processing to support handover is illustrated in Fig. 3.

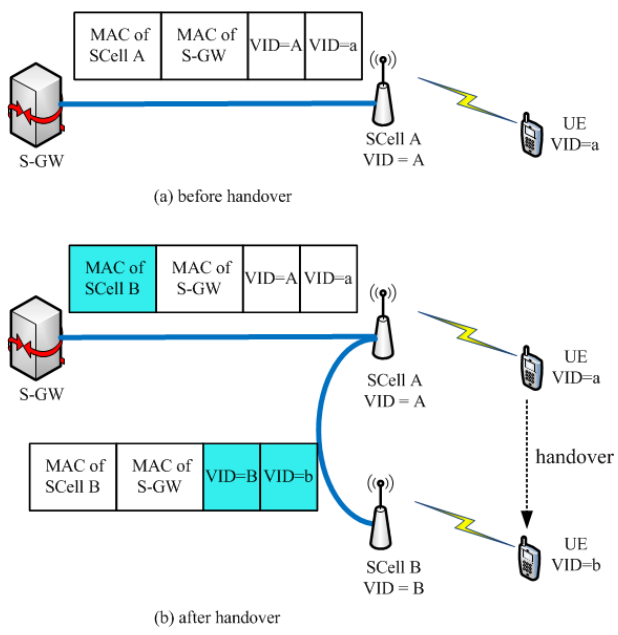

Fig. 3. Illustration of localized forwarding

Obviously, the above procedure just requires two signalling messages between the SDN controller and the switches: one for source SCell and the other for target SCell. Comparing to traditional policy, the number of required signalling messages is dramatically decreased. It should be admitted that such kind of localized forwarding may increase the end-to-end communication latency and consume the local resource. Therefore a threshold of the forwarding chain should be defined to balance the tradeoff between path switch cost and traffic forwarding cost. Also the localized forwarding chain between original serving SCell and the current serving SCell for an ongoing session can be selected according to the similar balance rule. There are mainly two candidates as follows:

\section{A. Cascaded Chain}

In this scheme, the localized forwarding chain between the original serving SCell and current serving SCell is formed by simply cascading the SCells along the trajectory of the UE, as long as the length of the chain does not exceed a predefined threshold. The threshold can be defined based on multiple metrics, such as end-to-end delay and hops, etc. If the threshold of the forwarding chain is exceeded, the SDN controller will setup a new forwarding path between S-GW and the target SCell, and release the old one.

\section{B. Shortest Chain}

In this scheme, the original SCell is regarded as a temporary anchor. In the handover completion phase, the SDN controller would select a path as the localized forwarding chain, which is the shortest one from the anchor SCell to current serving SCell. Thanking for the global view of SDN network, the SDN controller would be able to setup a new localized forwarding chain and release the old forwarding chain with the minimum control messages. Similar to the case of Cascade Chain, if the length of the shortest forwarding chain exceeds the predefined threshold, the SDN controller would setup the new forwarding path between S-GW and current serving SCell, and release the old one. To extend this scheme, actually during the whole session, the SDN controller can select any intermediate SCell in the forwarding chain as the temporary anchor, then setup the new chain from the anchor SCell to current serving SCell while keeping the chain part from the original SCell to anchor SCell unchanged.

\section{AnAlytical Model}

In this section, the performance of our proposal with Shortest Chain scheme is analysed in a grid-based SCell network as proposed in [12], [14]. It is difficult to model the Cascade Chain scheme since it is strongly dependent on the UE trajectory. In this network, each SCell is considered as a grid with four neighbors, as shown in Fig. 4. The UE can randomly move from the current cell to one of its four neighbors with equal probability. Each grid is indicated with a state variable $S_{i}^{(j)}(1 \leq i \leq K, 1 \leq j \leq 4 i)$ where $i$ is the tier of the grid related to the temporary anchor grid (i.e. original SCell denoted by $S_{0}^{(1)}$ ), $j$ is the grid index in its tier, and $K$ is the threshold of the forwarding chain.

The evolution of a UE's activity is modelled as a stochastic process that occurs in a sequence of discrete steps, which can be further analysed with a Discrete-Time Markov Chain (DTMC). Considering the fact that the threshold of the forwarding chain is limited, generally less than 10 , the approaching of state aggregation [12][14] does not applied in this analysis for the sake of simplicity. Therefore, the DTMC 


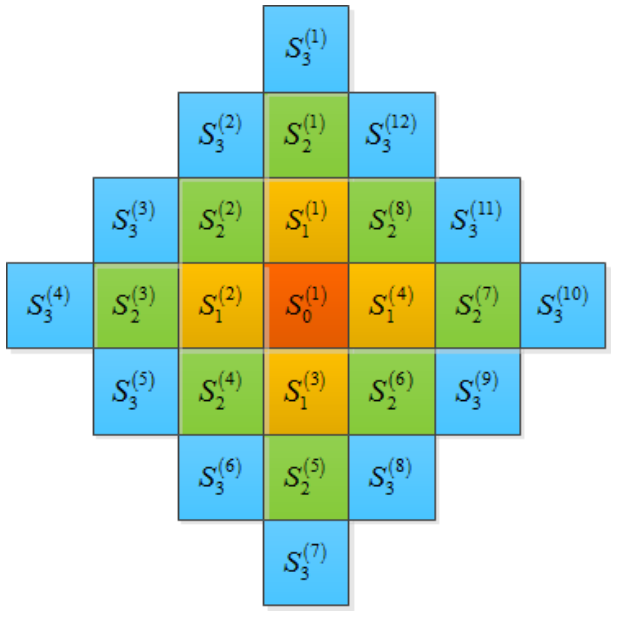

Fig. 4. Grid SCell network

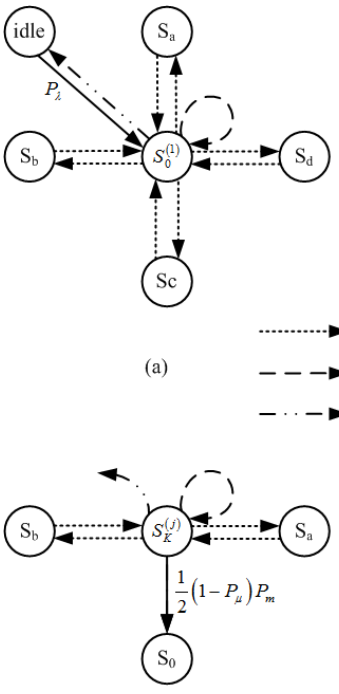

(c) $\bmod (j, K) \neq 1$

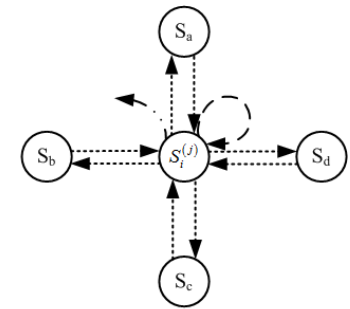

(b)

$1-P_{4}-\left(1-P_{4}\right) P$

$P_{\mu}$ (towards state 'idle')

Fig. 5. State transition diagrams

model for all states can be represented by four categories as shown in Fig. 5.

Among them, the state $S_{\text {idle }}$ denotes the state at which a UE has no ongoing sessions. Fig. 5(a) describes the transit behaviour of central grid, i.e. temporary anchor with $i=0$. Fig. 5(b) indicates the transit behaviour of grids with $i \in(0, K)$, and Fig. 5(c) (d) are for the grids in outermost ring with $i=K$. Here $S_{a}, S_{b}, S_{c}, S_{d}$ are designed to denote the four neighbours of certain grid. In this DTMC model, the UE changes state only at the end of each time slot $\Delta t$. Assuming that the sessions of a UE arrive as a Poisson process with rate $\lambda$, the duration of a session has an exponential distribution with mean $1 / \mu$, and the cell residence time also has an exponential distribution with mean $1 / m$, we obtain

$$
P_{\lambda}=\lambda \cdot \Delta t \quad P_{\mu}=\mu \cdot \Delta t \quad P_{m}=m \cdot \Delta t
$$

where $P_{\lambda}, P_{\mu}$ and $P_{m}$ are the probabilities of session arriving, session departure and a UE moving to another cell, respectively. Let $\pi_{i d l e}$ and $\pi_{i}^{(j)}(0 \leq i \leq K, 1 \leq j \leq 4 i)$ denote the stationary probability distribution of the UE in states $S_{i d l e}$ and $S_{i}^{(j)}$, respectively. Based on Fig. 5, the balance equations can be derived as in (1)-(5), as follows.

$$
\begin{aligned}
& \pi_{i d l e}=\left(1-P_{\lambda}\right) \pi_{i d l e}+P_{\mu} \pi_{0}^{(1)}+P_{\mu} \sum_{i=1}^{K} \sum_{j=1}^{4 i} \pi_{i}^{(j)} \\
& \left\{\begin{aligned}
\text { if } K= & 1 \\
\pi_{0}^{(1)}= & \left(1-P_{\mu}-\left(1-P_{\mu}\right) P_{m}\right) \pi_{0}^{(1)}+P_{\lambda} \pi_{i d l e}+ \\
& \left(1-P_{\mu}\right) P_{m} \sum_{h=\{a, b, c, d\}} \pi_{h}(0,1) \\
\text { if } K> & 1 \\
\pi_{0}^{(1)}= & \left(1-P_{\mu}-\left(1-P_{\mu}\right) P_{m}\right) \pi_{0}^{(1)}+P_{\lambda} \pi_{i d l e}+ \\
& \frac{1}{4}\left(1-P_{\mu}\right) P_{m} \sum_{h=\{a, b, c, d\}} \pi_{h}(0,1)+ \\
& \left(1-P_{\mu}\right) P_{m}\left(\frac{1}{2} \sum_{\substack{\bmod (j, K) \\
\neq 1}} \pi_{K}^{(j)}+\frac{3}{4} \sum_{\bmod (j, K)}^{=1} \pi_{K}^{(j)}\right)
\end{aligned}\right.
\end{aligned}
$$

$\forall 1 \leq i \leq K-1$ and $\forall 1 \leq j \leq 4 i$

$$
\begin{aligned}
\pi_{i}^{(j)}= & \left(1-P_{\mu}-\left(1-P_{\mu}\right) P_{m}\right) \pi_{i}^{(j)}+ \\
& \frac{1}{4}\left(1-P_{\mu}\right) P_{m} \sum_{h=\{a, b, c, d\}} \pi_{h}(i, j)
\end{aligned}
$$

$$
\left\{\begin{aligned}
(K= & 1 \text { and } \forall 1 \leq j \leq 4 K) \text { or } \\
(K> & 1 \text { and } \forall 1 \leq j \leq 4 K \text { and } \bmod (j, K)=1) \\
\pi_{K}^{(j)}= & \left(1-P_{\mu}-\left(1-P_{\mu}\right) P_{m}\right) \pi_{K}^{(j)}+ \\
& \frac{1}{4}\left(1-P_{\mu}\right) P_{m} \pi_{a}(K, j) \\
(K> & 1 \text { and } \forall 1 \leq j \leq 4 K \text { and } \bmod (j, K) \neq 1) \\
\pi_{K}^{(j)}= & \left(1-P_{\mu}-\left(1-P_{\mu}\right) P_{m}\right) \pi_{K}^{(j)}+ \\
& \frac{1}{4}\left(1-P_{\mu}\right) P_{m}\left(\pi_{a}(K, j)+\pi_{b}(K, j)\right)
\end{aligned}\right.
$$

$\pi_{i d l e}+\pi_{0}^{(1)}+\sum_{i=1}^{K} \sum_{j=1}^{4 i} \pi_{i}^{(j)}=1$

By solving (1)-(5), the stationary probability distributions and can be derived, which can be used to derive the performance metrics related to handover. TABLE I summarizes

TABLE I

PARAMETERS USED IN ANALYSIS

\begin{tabular}{r|l}
\hline \multicolumn{1}{|c|}{ Parameter } & Notation \\
\hline \hline$N_{b h}$ & $\begin{array}{l}\text { Hop number of backhaul forwarding path } \\
\text { between S-GW and SCell }\end{array}$ \\
\hline$D_{h o p}$ & $\begin{array}{l}\text { Transmission latency for one hop in } \\
\text { switch network }\end{array}$ \\
\hline$D_{\text {switch }}$ & Processing latency for packet in switches \\
\hline$\alpha$ & $\begin{array}{l}\text { Overlap factor of backhaul forwarding paths } \\
\text { before and after path switch,range is }(0,1)\end{array}$ \\
\hline
\end{tabular}

the parameters used in the following further analysis of two metrics including signalling cost and data delivery cost. 


\section{A. Signaling cost per UE}

Generally the path-switch signalling cost from the perspective of a single UE can be calculated based on the processing latency and the transmission latency. However, in SDN network, all the switches are centrally controlled by the SDN controller. To support path modification, the control messages will be transmitted from the SDN controller to switches, which will process these messages and apply the corresponding instructions. It is easy to conclude that all the related OpenFlow messages are transmitted and processed with the same way. Thus the signalling cost per UE can be evaluated by the number of required OpenFlow messages per UE. Referring to [12], the localized path switch for handover can be classified into four cases:

1) The 1st case: Here the localized forwarding chain is one of the shortest paths to the temporary anchor, and the forwarding chain will be cascaded. The number of required OpenFlow messages in this case is

$$
L_{1}=2
$$

2) The 2nd case: If the UE moves backward to the previous SCell, there would be a loop chain if cascaded. So in this case the SDN controller can just remove the last hop of the current forwarding chain, and the number of required OpenFlow messages is

$$
L_{2}=2
$$

3) The 3rd case: With the information of the whole nework topology, the SDN controller may find there is a shorter path between the target SCell and temporary anchor, and it could update the forwarding chain to the shorter path. This situation occurs when the source SCell is on tier $i$ and the target SCell is on tier $i-1$. In a grid model, the number of required OpenFlow messages for this update is independent on the location of SCell, which is

$$
L_{3}=4
$$

4) The 4th case: If the threshold of the forwarding chain is exceeded, the SDN controller will setup the new forwarding path between S-GW and the target SCell, and delete the previous forwarding path. Also suppose the overlap factor between the new forwarding path and the previous one is $\alpha$, the number of required OpenFlow messages is given as

$$
L_{4}=(2-\alpha) \times\left(N_{b h}+1\right)+K
$$

Thus we can define a vector $L=\left[L_{1}, L_{2}, L_{3}, L_{4}\right]^{T}$. In addition, a matrix $W_{(i, j)}$ is defined for SCell grid $S_{i}^{(j)}$ to indicate the handover cases to its four neighbors,

$$
W_{(i, j)}=\left[\begin{array}{cccc}
\omega_{11} & \omega_{12} & \omega_{13} & \omega_{14} \\
\omega_{21} & \omega_{22} & \omega_{23} & \omega_{24} \\
\omega_{31} & \omega_{32} & \omega_{33} & \omega_{34} \\
\omega_{41} & \omega_{42} & \omega_{43} & \omega_{44}
\end{array}\right]
$$

where $\omega_{m n}$ is 1 if the handover to the $m$-th $(1 \leq m \leq 4)$ neighbor is the $n$-th $(1 \leq n \leq 4)$ case. Otherwise, it is zero. Expect for the path-switch signalling cost for ongoing session, here we also take the signalling cost of session cease into account. The reason behind is that comparing to the traditional policy, our proposed localized forwarding policy may result in a longer forwarding path, which means the increase of signalling cost on flow entry deletion when session terminates. Thus the two kinds of signalling cost can be calculated as

$$
\begin{aligned}
C_{\text {path-switch }}= & \pi_{0}^{(1)}\left(1-P_{\mu}\right) P_{m} L_{1}+ \\
& \sum_{i=1}^{K} \sum_{j=1}^{4 i}\left[\frac{1}{4} \pi_{i}^{(j)}\left(1-P_{\mu}\right) P_{m} \psi_{(i, j)}\right]
\end{aligned}
$$

where $\psi_{(i, j)}=\operatorname{sum}\left(W_{(i, j)} \times L\right)$

$$
\begin{aligned}
C_{\text {session-cease }}= & P_{\mu}\left[\pi_{0}^{(1)}\left(N_{b h}+1\right)+\right. \\
& \left.\sum_{i=1}^{K} \sum_{j=1}^{4 i} \pi_{i}^{(j)}\left(N_{b h}+1+i\right)\right]
\end{aligned}
$$

By setting the forwarding chain threshold to be null, the path-switch signalling cost for traditional policy can be also derived.

\section{B. Data delivery cost per $U E$}

For an ongoing session, its packets would be delivered from S-GW to SCell along the whole forwarding path. Thus the expected total data delivery cost per UE can be expressed as

$$
\begin{aligned}
D= & \pi_{0}^{(1)}\left[N_{b h} D_{h o p}+\left(N_{b h}+1\right) D_{\text {switch }}\right]+ \\
& \sum_{i=1}^{K} \sum_{j=1}^{4 i} \pi_{i}^{(j)}\left[\left(N_{b h}+i\right) D_{h o p}+\left(N_{b h}+1+i\right) D_{\text {switch }}\right]
\end{aligned}
$$

\section{Numerical Results}

In this section, the proposed forwarding scheme is evaluated based on the performance metrics in section IV. The system parameter values used in this evaluation are provided in TABLE II. As shown in Fig. 6, we provided results by comparing our proposed scheme with traditional path-switch policy (i.e. switching the forwarding path as a whole for each handover) in the SDN-integrated backhaul network.

TABLE II

PARAMETER SETTING

\begin{tabular}{c|c|c|c}
\hline Parameter & Value & Parameter & Value \\
\hline \hline$\lambda$ & $0.01 / \min$ & $K$ & {$[1,2, \cdots, 8]$} \\
$\mu$ & $0.1 / \min$ & $N_{b h}$ & {$[5,8,10,15]$} \\
$m$ & $1 /$ min & $D_{\text {hop }}$ & 15 us \\
$\Delta t$ & 0.001 min & $D_{\text {switch }}$ & $15 u s$ \\
$\alpha$ & 0.8 & & \\
\hline
\end{tabular}

Fig. 6(a) shows the results in terms of the relative pathswitch signalling cost (RPSSC) ratio, where we observe that the signalling cost for path switch can be drastically decreased if the localized forwarding scheme applied, which is more than $50 \%$ but gradually convergent with the increase of the forwarding chain threshold (because of smaller probability of long forwarding chain for a particular traffic session). In another aspect, we can also see with the parameter $\mu$ decreasing, which means the traffic session will last longer, 



Fig. 6. Performance Vs. forwarding chain threshold $K$

the saving of path-switch signalling cost also decreases. The reason is that in this case it is more likely for the forwarding chain to exceed the threshold, thus be updated as a whole.

Fig. 6(b) indicates the combined performance including forwarding path switch and forwarding path deletion, so called relative combined signalling cost (RCSC) ratio. In this case, although the side effect of our proposal with longer forwarding path deletion, the overall gain on signalling cost saving is still achieved with our proposed scheme. Different from Fig. 6(a), the overall saving gain can be further exploited for the traffic session with longer duration. This is due to the factor that most attempts of forwarding path deletion are avoided with our proposal applied.

With the saving on signalling cost, the data delivery delay will be increased as shown in Fig. 6(c) with the metric of relative data delivery cost (RDDC) ratio. The larger the forwarding chain threshold is, the more time the delivery of data packet requires. Also the duration property of a session has the same effect on this data delivery cost as chain threshold. However, this minor increase on data delivery cost is allowable at most time (e.g. short forwarding chain), and even preferred for those applications with delay-jitter sensitivity. Additionally, in our simulation, the performance of backhaul is just simplified and abstracted as hop number while the realistic backhaul network is much more complex. If the backhaul latency is much higher than the local forwarding latency, which may be due to large hop number or congestion, the introduced extra delivery cost of our proposal will be much smaller (even negligible), which is partially demonstrated in Fig. 6(d) with the variation of backhaul hop $(\mathrm{BH})$ number.

\section{CONCLUSION}

In this paper, with respect to the integration of SDN and LTE mobile backhaul network, we designed a framework for traffic forwarding between S-GW and SCell under the concept of SDN network. A localized forwarding method is proposed for the mobility management, with the DTMCbased analytical model developed for performance evaluation.
Based on the numerical results, compared with traditional path-switch policy, significant signalling cost saving can be achieved with acceptable degradation on data delivery delay. In our future work, we will carry on further simulations and analytical comparisons with realistic system deployments and UE behaviors, and also investigate the mobility management scheme to support multiple sessions per UE.

\section{ACKNOWLEDGMENT}

The research leading to these results has received funding from the European Union Seventh Framework Programme (FP7/2007-2013) under grant agreement No.317941. The authors would like to acknowledge the contributions of their colleagues in iJOIN, although the views expressed are those of the authors and do not necessarily represent the project.

\section{REFERENCES}

[1] V. Jungnickel, K. Manolakis, W. Zirwas, etc. "The role of small cells, coordinated multipoint, and massive MIMO in 5G", IEEE Communications Magazine, Vol.52, No.5, pp. 44-51, May 2014.

[2] N. Bhushan, Junyi Li, D. Malladi, R. Gilmore, etc. "Network densification: the dominant theme for wireless evolution into 5G", IEEE Communications Magazine, Vol.52, No.2, pp.82-89,February 2014.

[3] I. Hwang, B. Song, S. Soliman, "A holistic view on hyper-dense heterogeneous and small cell networks", IEEE Communications Magazine, Vol.51, No.6, pp.20-27, June 2013.

[4] ONF, OpenFlow Switch Specification, V.1.4.0, Oct. 14, 2013

[5] K. Yap, M. Kobayashi, R. Sherwood, T. Huang, M. Chan, N. Handigol and N. Mckeown, "Openroads: empowering research in mobile networks”, SIGCOMM Computer Communication, Vol.40, pp.125-126, January 2010.

[6] C.J. Bernardos, A. De La Oliva, P. Serrano, etc., "An architecture for software defined wireless networking", IEEE wireless communications, Vol.21, No.3, pp.52-61, June 2014.

[7] H. Ali-Ahmad, C. Cicconetti, A. de la Oliva, etc., "An SDN-based network architecture for extremely dense wireless networks", 2013 IEEE SDN for Future Networks and Services, pp: 1-7.

[8] J. Costa-Requena, "SDN integration in LTE mobile backhaul networks", International Conference on Information Networking (ICOIN), Phuket, Thailand, pp.64-269, February 2014.

[9] A. Basta, W. Kellerer, M. Hoffmann, K. Hoffmann, etc., "A virtual SDN-enabled LTE EPC architecture: a case study for S-/P-Gateways functions", Future Networks and Services (SDN4FNS), Trento, Italy, pp.17, November 2013.

[10] G. Sun, G. Liu, H. Zhang and W. Tan, "Architecture on mobility management in OpenFlow-based radio access networks", IEEE Global High Tech Congress on Electronics (GHTCE), Shenzhen, China pp.2027, November 2013.

[11] P. Bertin, S.S. Bonjour and J.M. Bonnin, "Distributed or centralized mobility",IEEE GLOBECOM, Honolulu, Hawaii, pp.1-6, December 2009.

[12] T.Guo, A. ul Quddus, N. Wang, and R. Tafazolli, "Local mobility management for networked Femtocells based on X2 traffic forwarding", IEEE Transactions on Vehicular Technology, Vol.62, No.1, pp.326-340, January 2013.

[13] iJOIN Project, "D4.1 Report on SotA and requirements for networklayer algorithms and network operation and management", http://www.ictijoin.eu/, November 2013,

[14] R. Balakrishnan and I.F. Akyildiz, "Local mobility anchoring for seamless handover in coordinated small cells", IEEE Globecom Workshops, Atlanta, GA, USA, pp.4498-4494, December 2013. 